\title{
Caution: Plasmid DNA topology affects luciferase assay reproducibility and outcomes
}

\author{
Emma Tudini, ${ }^{\ddagger, 2}$, Lez J Burke ${ }^{\ddagger, 1}$, Phillip J Whiley ${ }^{1,2}$, Jan Sevcik ${ }^{1,3}$, Amanda B Spurdle ${ }^{2}$ \& Melissa A Brown ${ }^{\star, 1}$
}

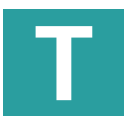

ransfection of cell lines with purified plasmid DNA is a wellestablished technique for analysis of gene and protein function, and gene regulation [1]. To ensure reproducibility and sensitivity of transfections, it is essential that assays are well controlled and reproducible. This is achieved by optimizing a number of factors, including the ratio of plasmid DNA and transfection reagents, the number of cells transfected, and the choice of cell line and transfection reagent. In addition, multiple biological and technical replicates and robust statistical analyses are crucial.

With the use of next-generation sequencing to identify genetic changes underlying disease risk, the number of potential disease-associated variants residing in noncoding regions of the genome has increased dramatically. Determining the functional and clinical significance of such variants is important in order to evaluate the risk such variants pose to variant carriers and provide accurate genetic counselling. Currently, in vitro reporter assays present the most commonly used analysis method for assessing activity of gene regulatory regions, whereby a regulatory element drives the expression of a reporter gene such as luciferase. The influence of

\section{KEYWORDS}

assays $\cdot$ cell culture $\cdot$ gene expression analysis - in vitro models • transfection

'School of Chemistry \& Molecular Biosciences, The University of Queensland, St Lucia, 4067 QLD, Australia; ${ }^{2}$ Department of Genetics \& Computational Biology, QIMR Berghofer Medical Research Institute, Herston, 4006 QLD, Australia; Institute of Biochemistry and Experimental Oncology, First Faculty of Medicine, Charles University, Prague, Czech Republic; *Author for correspondence: melissa.brown@uq.edu.au; $¥$ These authors contributed equally

BioTechniques 67: 94-96 (September 2019) 10.2144/ btn-2019-0060 particular DNA sequence variants on this regulatory activity vcan then be determined. Given that many variants that contribute to disease susceptibility have a subtle effect on gene regulation, it is essential that experimental assays are robust and reproducible. Here we show that results of a reporter assay commonly used for this purpose is significantly impacted by the topology of reporter plasmid DNA.

Single-nucleotide DNA sequence variants within the transcriptional promoter of the BRCA1 gene (hg19; chr17:4127718641278132 plus chr17:41276114-41276753) were analyzed for an effect on regulatory activity, using the pGL3 luciferase reporter system (Promega, WI, USA) [2]. To enable evaluation of the effect of the variants, an internal control (pGL3-promoter; Promega) was analyzed simultaneously. The internal control, a wild-type (WT) reporter plasmid, and reporter plasmids containing the variants, were prepared simultaneously and DNA concentration was determined by Nanodrop (ThermoFisher MA, USA). MCF7 breast cancer-derived cells seeded in 24-well plates were transfected with both the reporter plasmid and a transfection control plasmid containing a Renilla reporter sequence ( $\mathrm{pRL}$-TK; Promega) using Lipofectamine 3000 (Life Technologies, CA, USA). Luciferase activity was measured after $48 \mathrm{~h}$ [3] and relative activities of the variant $B R C A 1$ promoters were compared to the activity of the WT $B R C A 1$ promoter. Biological replicates were generated using independent batches of cells cultured and transfected at separate times with independently prepared sets of plasmids. All reporter luciferase activities were normalized to Renilla activity, and subsequently to the internal control, which allowed calculation of the variance of the WT reporter. Technical replicates were averaged and treated as a single biological replicate, and statistics performed on the biological replicates [2].
During these analyses, we noticed considerable variability in our reporter assay data (Figure 1A). Analysis of the purified plasmid DNA on agarose gels revealed differently migrating DNA species. The electrophoretic properties of the plasmids were consistent with well-established topologies of plasmid DNA, specifically supercoiled DNA (fast migrating) and nonsupercoiled DNA (slow migrating) [4-6].

To analyze the effect of differences in the topology of plasmid DNA on luciferase reporter activity, multiple sets of plasmids were purified for a number of different reporter constructs, each containing different variants in the $B R C A 1$ promoter. Supercoiled and nonsupercoiled versions of each plasmid were then used to transfect cells, and luciferase activity was determined relative to a supercoiled control plasmid, purified at the same time. When all variant-containing plasmids were supercoiled, none of the variants had an effect on the reporter activity relative to WT (Figure 1B). However, when plasmids that were not supercoiled were compared to the supercoiled WT reporter, four of the variants displayed a significant effect on reporter activity (Figure 1C). Thus, plasmid topology has a significant impact on reporter activity and leads to over-reporting of an impact on regulatory activity.

These results are consistent with previous reports showing that the conformation of plasmids impacts the results of other cell-based assays $[4,5,6]$. As such, it is imperative to ensure that the topology of the transfected plasmid DNA is consistent before interpreting the differences in luciferase activity for different nucleotide sequences. Failure to do so may result in functional activity being undetectable in the case of activating variants, or interpreted as having a significant effect where the variant, in fact, has no effect on reporter activity. 


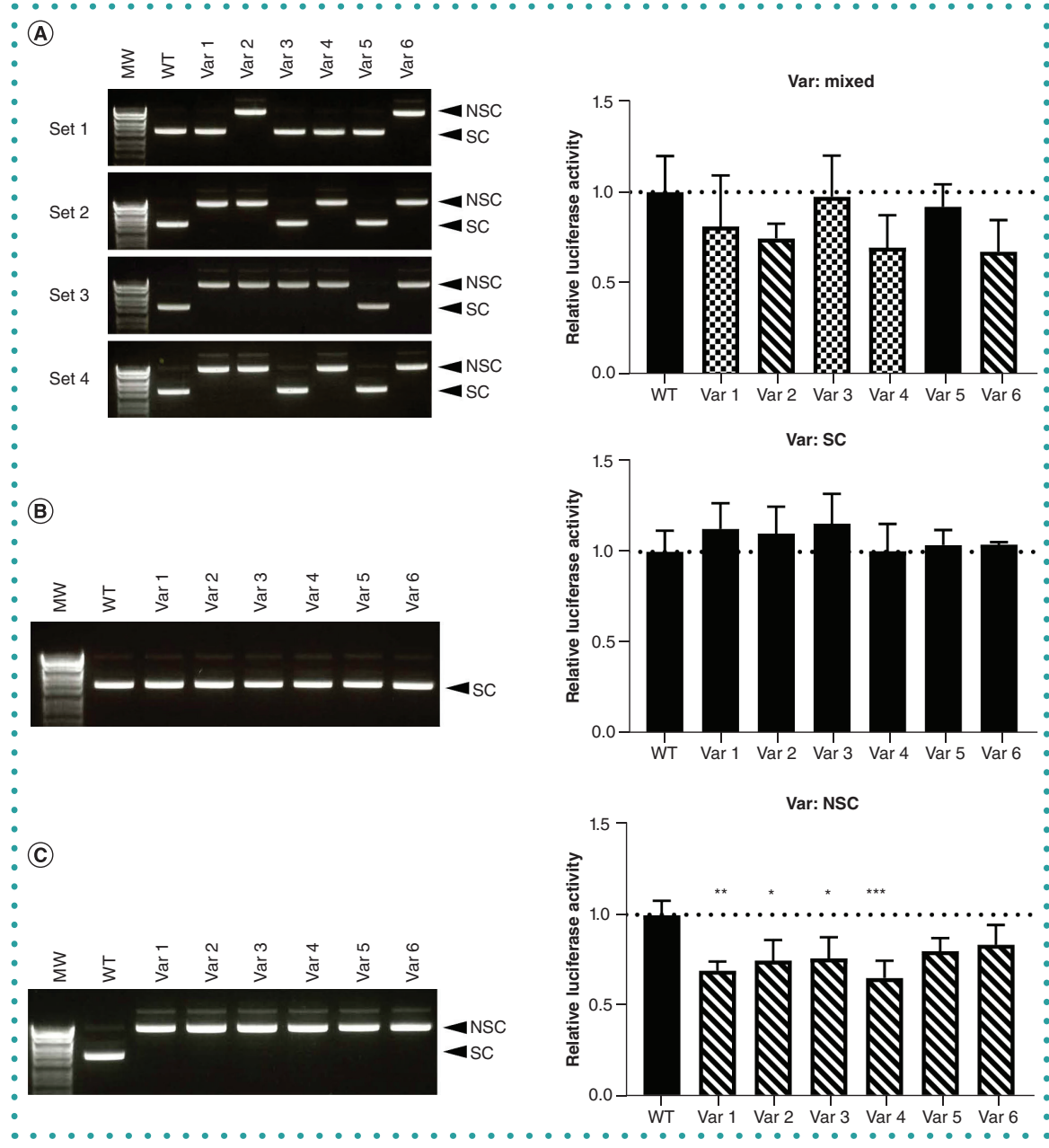

Figure 1. Plasmid topology can influence luciferase activity in transient transfections. (A) Gel electrophoresis (left panel) and luciferase activity in MCF7 cells (right panel) of four independent preparations (set 1-4) of plasmid DNA (pGL3-basic backbone) containing an active portion of the BRCA1 promoter driving the luciferase gene with the WT promoter or single nucleotide variants (Var 1-6). Plasmids $(200 \mathrm{ng})$, prepared using Qiagen miniprep kits, were separated on 1\% TAE agarose gels. Migration position of plasmids that are SC and NSC is indicated. MW: 1 kb DNA ladder (Bioline, TN, USA). Luciferase activity is shown relative to the activity of the supercoiled WT plasmid. The horizontal dotted line represents relative WT promoter activity set at 1.0-fold. Data represent the average \pm standard deviation of four independent transfections of independently plated MCF7 cells using plasmid sets prepared at different times. Data are shown from plasmids that are SC (black bars), NSC (striped bars) or a mixture of topologies (checked bars). (B \& C) Gel electrophoresis of representative independent plasmid preparations (left panel) and associated luciferase activity (right panel) for SC WT and single-nucleotide variantcontaining plasmids that were classified as SC (B) or NSC (C). Relative luciferase activity was compared with the WT SC plasmid using an ANOVA followed by Tukey's post-hoc test in GraphPad Prism. * $\mathrm{p}<0.05 ; * * \mathrm{p}<0.01 ; * * * \mathrm{p}<0.005$.

MW: Molecular weight; NSC: Not supercoiled; SC: Supercoiled; WT: Wild-type.

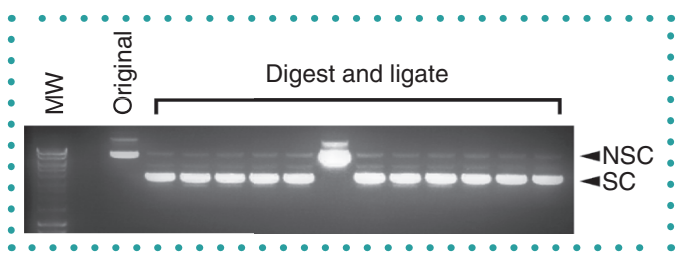

- The mechanism underlying the impact of plasmid topology on reporter activity is unknown. Comparison of DNA concentration by absorbance at $260 \mathrm{~nm}$ and electrophoresis on agarose gels (Figure 1) suggests that is it not caused by changes in the measurable amount of DNA. In addition, all plasmid DNA preparations, regardless of topology, were of similar purity (as determined by Nanodrop) and contained no protein and/or other contaminants as indicated by A260/A280 or A260/A230 ratios, that would contribute to the observed results. A more plausible hypothesis is that DNA topology affects transfection and expression of the plasmid. Consistent with this, previous studies have shown that supercoiled plasmids drive higher levels of expression and thus the activity of expressed products, using other transfection methods such as DEAE dextran [4], liposomal DOTAP/DOPE complexes [5] and cationic polymers [6]. A potential mechanism for this may be that nonsupercoiled plasmids have a reduced transfection rate impacting the availability of plasmid DNA for transcription. While we have shown the relevance of plasmid DNA topology for luciferase assays, it would be prudent to consider the impact of plasmid topology for any cell-based functional assays that rely on transfection. Based on this, we recommend that only plasmids that have been confirmed to have a supercoiled topology be used in transfection assays.

The conformation of the plasmid DNA is often retained after transformation and repurification; that is, a nonsupercoiled plasmid DNA will give rise to predominantly nonsupercoiled plasmid DNA after repreparation. This hinders purification of supercoiled plasmids from preparations that are not supercoiled. Here we show that digestion of a nonsupercoiled plasmid containing a 950-base pair BRCA1 promoter (hg19; chr17:4127718641278132) insert with a restriction enzyme that cuts once in the plasmid and religation, results in purification of supercoiled plasmids in 10/11 cases (Figure 2). This procedure allows purification of supercoiled plasmids

Figure 2. Supercoiled conformation can be restored by digestion and religation. A plasmid (NSC) containing an active portion of the BRCA1 promoter was digested with Sall, ligated and used to transform Escherichia coli from which plasmid DNA was repurified. Agarose gel showing original plasmid (200 ng) that is not SC (original) and $1 \mu \mathrm{l}$ of plasmid minipreps after digestion, ligation and retransformation. Migration position of plasmids that are SC and NSC is indicated, showing restoration on 10/11 occasions. MW: 1 kb DNA ladder (Bioline, TN, USA). MW: Molecular weight; NSC: Not supercoiled; SC: Supercoiled. 
from plasmids that were previously not supercoiled, and thus for experiments to be controlled for plasmid topology.

In summary, we have identified another factor that can affect the outcome of luciferase reporter assays of sequence variants mapping to gene regulatory regions. We recommend routine assessment of plasmid topology and the use of only plasmids that are supercoiled for transfection. Good laboratory practice would further suggest that all reporter plasmids to be assayed are prepared simultaneously, that test and control plasmids are analyzed on the same plate, and that a minimum of three technical and three biological replicates are performed for each experiment.

\section{SUPPLEMENTARY DATA}

To view the supplementary data that accompany this paper please visit the journal website at: www.future-science. com/doi/suppl/10.2144/btn-2019-0060

\section{AUTHOR CONTRIBUTIONS}

$E T, L J B, A B S$ and $M A B$ conceived and designed the study. ET, LJB, JS and PJW performed the experiments. ET, LJB, JS, $A B S$ and $M A B$ wrote and revised the manuscript.

\section{FINANCIAL \& COMPLETING INTERESTS DISCLOSURE}

This work was supported by grants from the National Health and Medical Research Council (ID1104808) and Cancer Council Queensland (ID1044008 and ID1026095) to MA Brown, and the Ministry of Health of the Czech Republic (AZV 16-33444A) to J Sevcik. AB Spurdle is supported by an NHMRC Senior Research Fellowship (ID1061779). The authors have no other relevant affiliations or financial involvement with any organization or entity with a financial interest in or financial conflict with the subject matter or materials discussed in the manuscript apart from those disclosed.

No writing assistance was utilized in the production of this manuscript.

\section{ETHICAL CONDUCT OF RESEARCH}

The authors state that they have obtained Institutional Biosafety committee (IBC) approval to undertake the described experiments.

\section{OPEN ACCESS}

This work is licensed under the AttributionNonCommercial-NoDerivatives 4.0 Unported License. To view a copy of this license, visit http://creativecommons.org/ licenses/by-nc-nd/4.0/

\section{REFERENCES}

1. Kim TK, Eberwine JH. Mammalian cell transfection: the present and the future. Anal. Bioanal. tion: the present and the future. A
Chem. 397(8), 3173-3178 (2010).

2. Burke LJ, Sevcik J, Gambino $\mathrm{G}$ et al. BRCA1 and BRCA2 5 ' noncoding region variants identified in breast cancer patients alter promoter activity and protein binding. Hum. Mutat. 39(12), 2025-2039 (2018).

3. Brewster BL, Rossiello F, French JD et al. Identification of fifteen novel germline variants in the BRCAT 3UTR a functional miR-103 target site. Hum. Mutat. 33(12), 1665-1675 (2012).

4. Weintraub $\mathrm{H}$, Cheng PF, Conrad K. Expression of transfected DNA depends on DNA topology. Cell 46(1) 115-122 (1986)

5. Remaut K, Sanders NN, Fayazpour F, Demeester J, De Smedt SC. Influence of plasmid DNA topology on the transfection properties of DOTAP/DOPE lipoplexes. $J$. Control Release 115(3), 335-343 (2006).

6. Cherng JY, Schuurmans-Nieuwenbroek NM, Jiskoot $W$ et al. Effect of DNA topology on the transfection efficiency of poly((2-dimethylamino)ethyl methacrylate)-plasmid complexes. J. Control Release 60(23), 343-353 (1999).

\section{5}

microplate washer

\section{Set it. Runit. Forget it.}

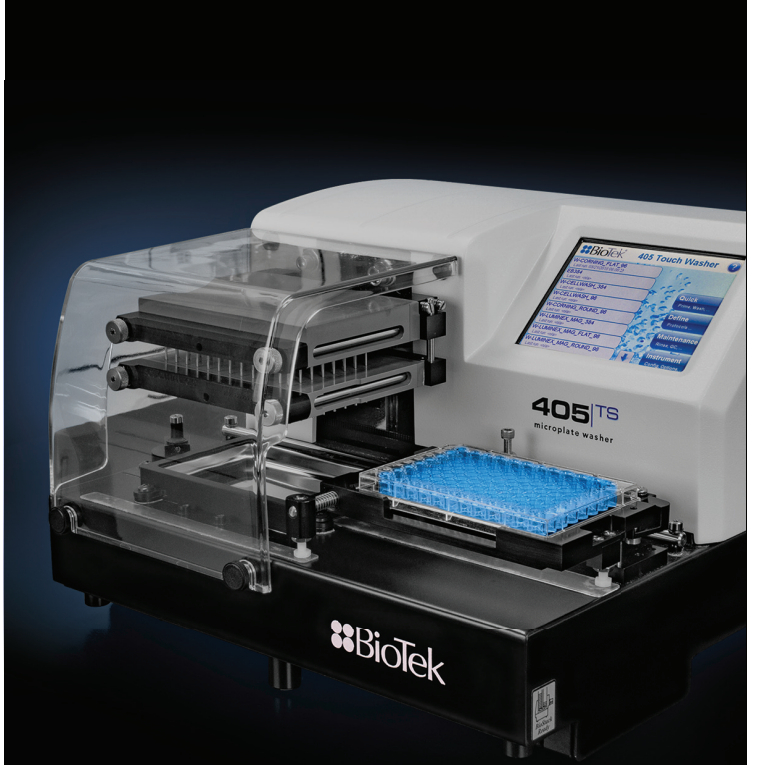

\section{BioTek. \\ \#1 in plate washing.}

Now easier to use and

more powerful than ever.

Programmable residual

volume, protocol locking,

enhanced vacuum

adjustment for cell-based

assays, and more.

www.biotek.com/405TS

Think Possible

zaBioTek 